\title{
Time-resolved multiwavelength observations of the blazar VER J0521+211 from radio to gamma-ray energies
}

\author{
H. Prokoph ${ }^{* a}$ for the VERITAS Collaboration ${ }^{\dagger}$ \\ ${ }^{a}$ Department of Physics and Electrical Engineering, Linnaeus University, 35195 Växjö, Sweden \\ E-mail: heike.prokophelnu.se
}

\section{P. Da Vela ${ }^{b}$ and C. Schultz ${ }^{c}$ for the MAGIC Collaboration}

${ }^{b}$ Università di Siena and INFN Pisa, I-53100 Siena, Italy

${ }^{c}$ Università di Padova and INFN, I-35131 Padova, Italy

\begin{abstract}
VER J0521+211 (RGB J0521.8+2112) is one of the brightest and most powerful blazars detected in the $\mathrm{TeV}$ gamma-ray regime. It is located at a redshift of $z=0.108$ and since its discovery in 2009, VER J0521+211 has exhibited an average TeV flux exceeding 0.1 times that of the Crab Nebula, corresponding to an isotropic luminosity of $3 \times 10^{44} \mathrm{erg} \mathrm{s}^{-1}$. We present data from a comprehensive multiwavelength campaign on this object extending between November 2012 and February 2014, including single-dish radio observations, optical photometry and polarimetry, UV, $\mathrm{X}$-ray, $\mathrm{GeV}$ and TeV gamma-ray data (VERITAS, MAGIC). Significant flux variability was observed at all wavelengths, including a long-lasting high state at gamma-ray energies in Fall 2013. Nightly-resolved spectra at X-ray and $\mathrm{TeV}$ energies are be presented, and emission mechanisms explaining the observed flux and spectral variability are discussed.
\end{abstract}

The 34th International Cosmic Ray Conference,

30 July- 6 August, 2015

The Hague, The Netherlands

\footnotetext{
* Speaker.

${ }^{\dagger}$ veritas.sao.arizona.edu

\#magic.mpp.mpg.de
} 


\section{Introduction}

VER J0521+211, spatially associated with the radio and X-ray source RGB J0521.8+2112, was discovered in 2009 as $\mathrm{TeV}$ emitter by VERITAS [1] and is now unambiguously classified as a BL Lac-type blazar [2]. As usually observed in blazars, VER J0521+211 is highly variable at all wavelengths. With an average integral flux above $200 \mathrm{GeV}$ of about $>0.1 \mathrm{Crab}^{1}$, VER J0521+211 is among the brightest known TeV blazars. Located at a redshift of $z=0.108$ [4], this flux corresponds to a gamma-ray luminosity of about $3 \times 10^{44} \mathrm{erg} \mathrm{s}^{-1}$ and is thus significantly brighter than the classical northern high-synchrotron-frequency-peaked BL Lacs (HBLs) such as Mrk 421, Mrk 501 and 1ES 1959+650. VER J0521+211 itself shows a synchrotron peak in the optical regime, suggesting a classification as intermediate-frequency-peaked BL Lac (IBL). However, during the TeV high state in November 2009 [5], VER J0521+211 showed more HBL-like properties, especially given the observed spectral hardening in the X-ray regime with increased flux. The observed variability and its bright $\mathrm{TeV}$ flux motivated further multiwavelength observations of VER J0521+211 in order to extend the detailed time-resolved spectral modeling available for nearby HBLs $[6,7,8]$ to a more luminous blazar which are reported here.

\section{VERITAS}

VERITAS is an array of four $12 \mathrm{~m}$-diameter imaging atmospheric Cherenkov telescopes (IACTs), located in southern Arizona, USA. It is sensitive to gamma-ray energies between $85 \mathrm{GeV}$ and $>30 \mathrm{TeV}$ and is capable to detect a source with $0.01 \mathrm{Crab}$ flux in under $\sim 25$ hours of observations. VERITAS observations of VER J0521+211 reported here were taken in two seasons: the 2012 season comprising data taken between 2012 Nov 11 and 2013 Feb 14; and the 2013 season (2013 Oct 15 to 2014 Feb 01). All data were taken in wobble mode for which the source is observed with an offset of $0.5^{\circ}$ with respect to the center of the instrument's field of view to allow for simultaneous background measurements [9]. After quality selection, the dataset comprised $5.0 \mathrm{~h}$ live time in the 2012 season and $16.5 \mathrm{~h}$ in the 2013 season. VERITAS data analysis followed the procedure outlined in [10]. Cherenkov light from air showers initiated by gamma rays and cosmic rays triggers the readout of PMT signals, which are then calibrated and used to reconstruct an image of the shower in the focal plane. Individual telescope images are parameterized by moment analysis, and geometrical reconstruction is used to estimate the arrival direction, $\theta$, of the primary particle with respect to the source location. The signal was extracted using a reflected region background model with an $\mathrm{ON}$ region of $0.17^{\circ}$ radius centered on the position of VER J0521+211 (R.A. $=05^{\mathrm{h}} 21^{\mathrm{m}} 45^{\mathrm{s}}$, Decl. $\left.=+21^{\circ} 12^{\prime} 51 .^{\prime \prime} 4, \mathrm{~J} 2000\right)$. The strength of the gamma-ray signal is evaluated using Eq. 17 in [11] and was found to be $19.0 \sigma$ in 2012 and $60.3 \sigma$ in 2013. The average flux levels in the two seasons are $F_{>0.2 \mathrm{TeV}}^{\prime 12}=2.4 \pm 0.2$ and $F_{>0.2 \mathrm{TeV}}^{\prime 13}=4.2 \pm 0.1$ in units of $10^{-11} \mathrm{~cm}^{-2} \mathrm{~s}^{-1}$. For comparison, the time averaged flux in [2] was $1.9 \pm 0.1$ in the same units (as shown in Figure 1).

\section{MAGIC}

MAGIC is a stereoscopic system of two 17 m-diameter IACTs, located at La Palma, Canary

\footnotetext{
${ }^{1}$ The gamma-ray flux of the Crab Nebula used here is $F(E>200 \mathrm{GeV})=2.1 \times 10^{-10} \mathrm{~cm}^{-2} \mathrm{~s}^{-1}[3]$.
} 

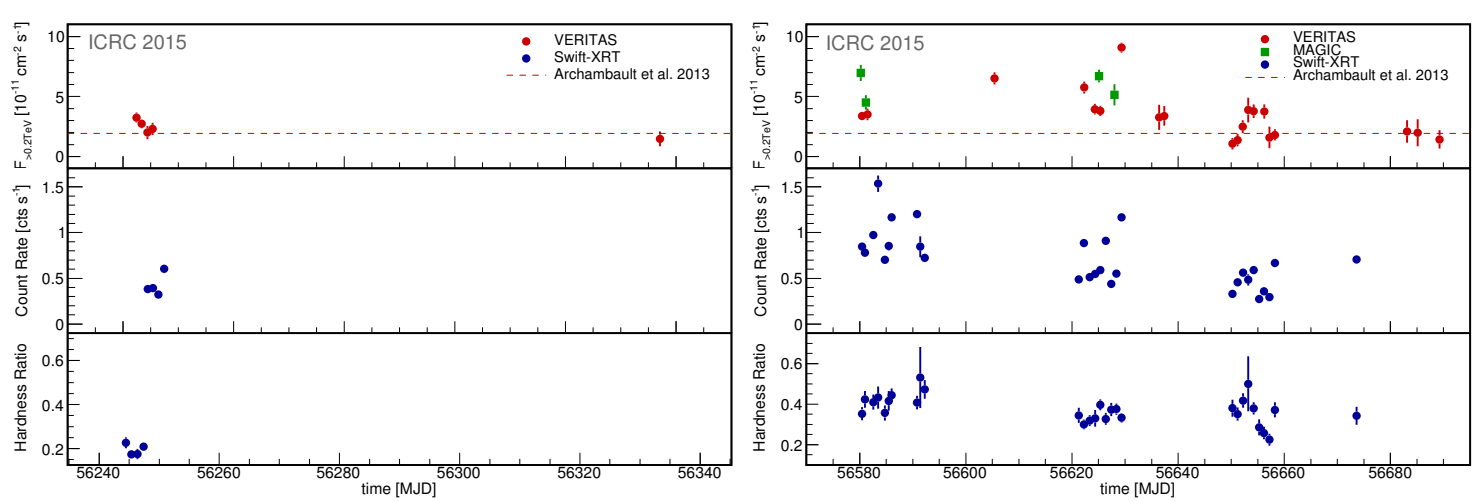

Figure 1: Evolution of the TeV flux, $\mathrm{X}$-ray flux, and $\mathrm{X}$-ray hardness ratio as a function of time during the 2012 (left) and 2013 seasons (right). It should be noted that the MAGIC and VERITAS observations in 2013 were not taken simultanously.

Islands. It is sensitive to gamma-ray energies above $\sim 50 \mathrm{GeV}$ (in normal trigger mode) and underwent a series of upgrades during summer 2011 and 2012 [12]. In stereoscopic mode, the integral sensitivity for sources with Crab Nebula-like spectra above $220 \mathrm{GeV}$ is $(0.66 \pm 0.03) \%$ of Crab Nebula flux in $50 \mathrm{~h}$ of observations (more details about the MAGIC performance are given in [13]). MAGIC observations of VER J0521+211 were taken between 2013 Oct 15 and Dec 2 and were triggered by the high state in optical and high-energy gamma rays reported by KVA (E. Lindfors, private communication) and Fermi-LAT [14], respectively. Observations covering a zenith angle range from $7^{\circ}$ to $26^{\circ}$ were performed in wobble mode with an offset of $0.4^{\circ}$. After quality selection and dead time correction, the total effective observation time was $\sim 4.5$ hours. The data were analyzed using the MAGIC analysis and reconstruction software package [15], which has been adapted to stereoscopic observations [16]. The signal was extracted from an ON region of $0.11^{\circ}$ radius centered on the position of VER J0521+211. The analysis of the overall data set yielded a strong signal of $30.5 \sigma$ significance [11], while the significances of the signal from the individual nights are $18.6 \sigma, 9.7 \sigma, 21.9 \sigma$ and $6.2 \sigma$ for Oct 15, 16, Nov 29 and Dec 2, respectively. Given the significance of the signal, night-wise spectra could be obtained above the energy threshold of $65 \mathrm{GeV}$ for the observations in October and November and are shown in Figure 2.

\section{X-ray}

The Swift X-ray telescope (XRT [17]) monitored VER J0521+211 in the X-ray band (0.3$10 \mathrm{keV}$ ) for most of the nights of the campaign. Data were processed using the most recent versions of the standard Swift tools: Swift Software version 3.9, FTOOLS version 6.12 and XSPEC version 12.7.1. Light curves are generated using xrtgrblc version 1.6. More details on the monitoring program and the analysis procedure can be found in [18].

\section{Flux and spectral variability}

The TeV fluxes measured in 2012 and 2013 are higher than those reported during the discovery of the source [2], with the average flux in 2013 being a factor of 2 brighter than that of 2009. The 


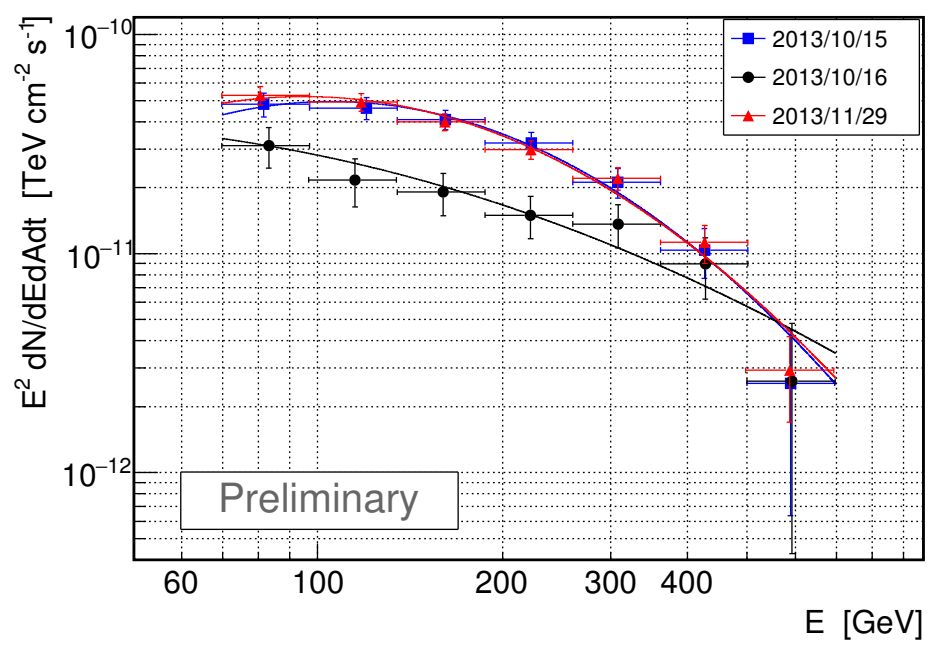

Figure 2: Spectral energy distribution of VER J0521+211 as obtained by MAGIC during three single nights in 2013.

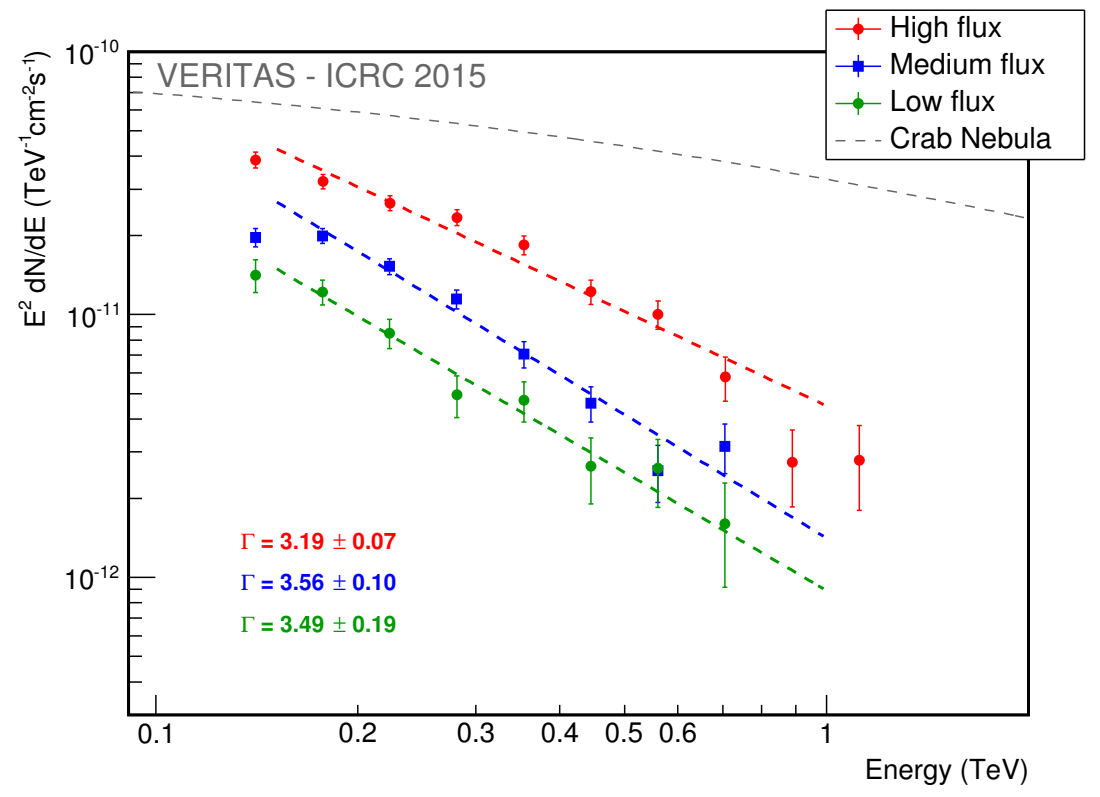

Figure 3: Comparison of the spectral energy distributions measured with VERITAS at different flux states. Spectra have been obtained by stacking observations with nightly fluxes $F_{>0.2 \mathrm{TeV}}<3 \times 10^{-11} \mathrm{~cm}^{-2} \mathrm{~s}^{-1}$ (low flux), $F_{>0.2 \mathrm{TeV}}>4 \times 10^{-11} \mathrm{~cm}^{-2} \mathrm{~s}^{-1}$ (high flux), and a medium flux bin defined between these two values. 
nightly flux (Figure 1) shows significant variability down to timescales of 1 day.

To test for spectral variability, we have produced gamma-ray spectra for individual observing nights (Figure 2) and by stacking observations at different flux levels (Figure 3). At low and medium flux levels we find gamma-ray spectra largely compatible with a power-law with index $\Gamma=3.44 \pm 0.20$ that was reported in previous observations [2]. Co-adding the spectra from the three nights with higher fluxes we measure $\Gamma=3.10 \pm 0.07$, constituting evidence for a mild hardening of the gamma-ray spectrum when the emission is brighter. In addition, the lower threshold of the MAGIC observations (Figure 2) helps to reveal some evidence of spectral curvature, suggesting a peak of the inverse-Compton emission component at energies of $\sim 100 \mathrm{GeV}$.

Similar levels of 1-day-scale flux variability can be seen in the X-ray band (Figure 1). The evolution of the hardness ratio in the X-ray band (Figure 4) suggests only small changes of the $\mathrm{X}$-ray spectral shape at different flux levels. Data from the nights with simultaneous observations by VERITAS and Swift-XRT (Figure 5) show a clear correlation between the measured fluxes in both bands.

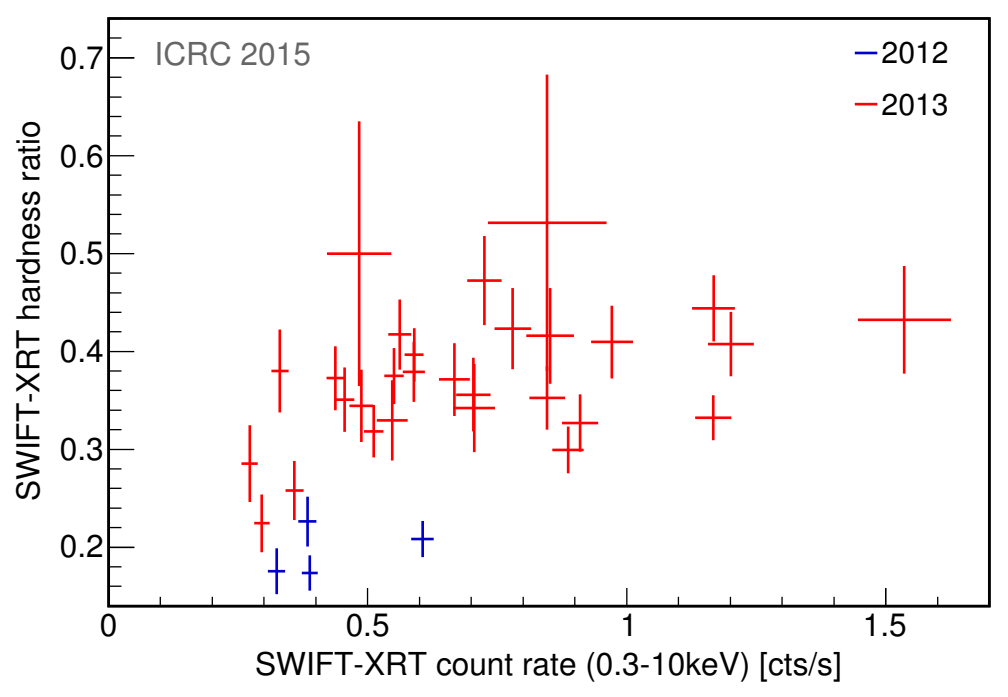

Figure 4: X-ray hardness ratio as a function of X-ray flux measured by Swift-XRT. The hardness ratio is defined as the rate in the $2-10 \mathrm{keV}$ band divided by the rate in the $0.3-2 \mathrm{keV}$ band.

\section{Discussion}

The data presented in these proceedings constitute the first extensive multi-wavelength campaign on VER J0521+211. Significant changes in the X-ray and TeV fluxes from the source were seen in timescales of $\sim 1$ day, with an amplitude of approximately a factor of 5 in both bands. Spectral variability in the $\mathrm{TeV}$ band is first reported here, displaying a mild hardening of the spectrum at higher flux levels. The spectral variability is however much less significant than typically found in HBLs.

The observed correlation between the fluxes in the $\mathrm{X}$-ray and $\mathrm{TeV}$ bands suggests a connection between the synchrotron and the high-energy spectral components of the emission, as expected if the high-energy emission is dominated by synchrotron self-Compton. However, a contribution 


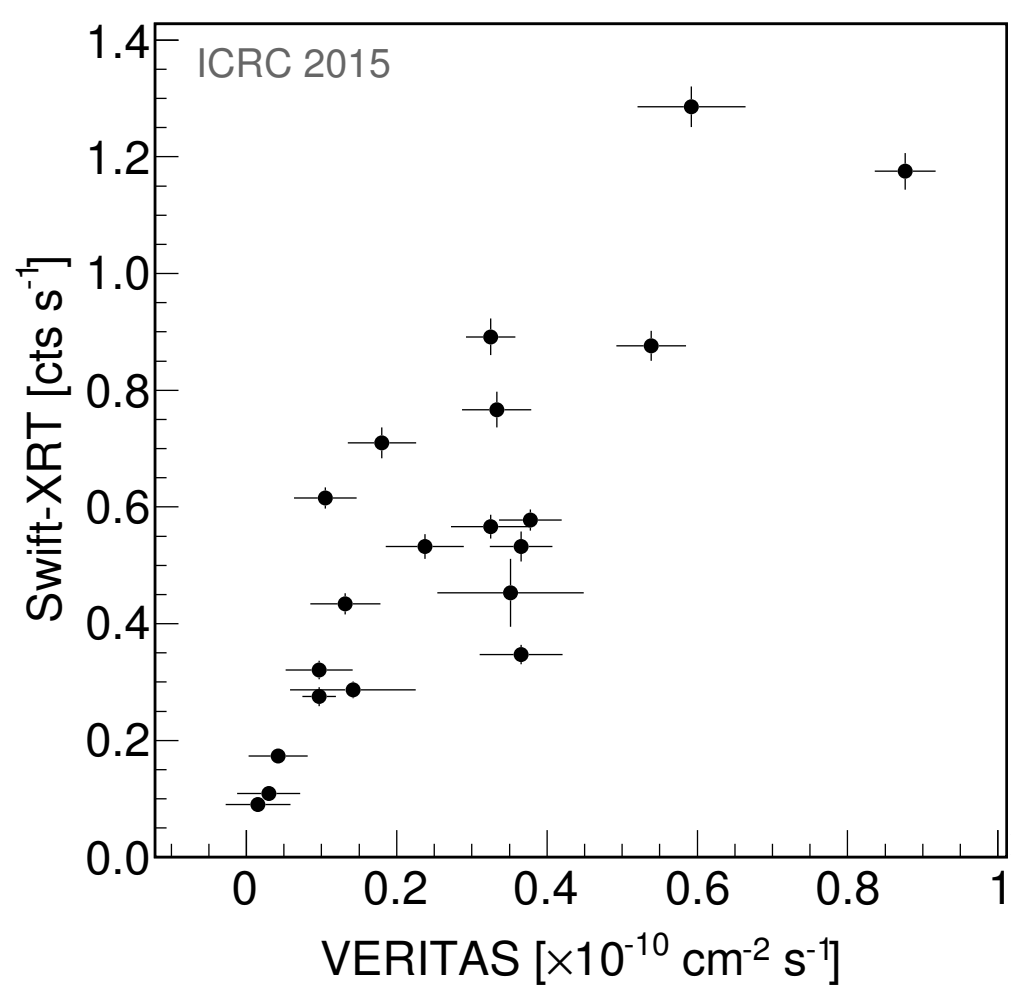

Figure 5: X-ray flux as a function of the TeV flux for nights with simultaneous observations in both bands.

from inverse-Compton emission from electrons and positrons interacting with other radiation fields external to the jet cannot be excluded.

Compared to similar studies conducted on TeV HBLs (Mrk 421, Mrk 501, 1ES 1959+650), the level of spectral variability in the $\mathrm{TeV}$ regime is small given the high-significance of the detections and the $\mathrm{TeV}$ flux changing by a factor of $\sim 5$. This small level of spectral variability and a softer spectral index seem characteristic of IBLs, where electrons and positrons with energies beyond the $\mathrm{TeV}$ scale will be less dominant than in HBLs given the higher rate of inverse-Compton cooling.

VERITAS is supported by grants from the U.S. Department of Energy Office of Science, the U.S. National Science Foundation and the Smithsonian Institution, and by NSERC in Canada. We acknowledge the excellent work of the technical support staff at the Fred Lawrence Whipple Observatory and at the collaborating institutions in the construction and operation of the instrument. The VERITAS Collaboration is grateful to Trevor Weekes for his seminal contributions and leadership in the field of VHE gamma-ray astrophysics, which made this study possible.

MAGIC would like to thank the Instituto de Astrofisica de Canarias for the excellent working conditions at the Observatorio del Roque de los Muchachos in La Palma. The financial support of the German BMBF and MPG, the Italian INFN and INAF, the Swiss National Fund SNF, the ERDF under the Spanish MINECO (FPA2012-39502), and the Japanese JSPS and MEXT is grate- 
fully acknowledged. This work was also supported by the Centro de Excelencia Severo Ochoa SEV-2012-0234, CPAN CSD2007-00042, and MultiDark CSD2009-00064 projects of the Spanish Consolider-Ingenio 2010 programme, by grant 268740 of the Academy of Finland, by the Croatian Science Foundation (HrZZ) Project 09/176 and the University of Rijeka Project 13.12.1.3.02, by the DFG Collaborative Research Centers SFB823/C4 and SFB876/C3, and by the Polish MNiSzW grant 745/N-HESS-MAGIC/2010/0.

\section{References}

[1] Ong, R. A., et al., VERITAS discovery of a new VHE Gamma-ray Source, VER J0521+211, The Astronomer's Telegram, 2260, 1 (2009)

[2] Archambault, S., Arlen, T., Aune, T., et al., Discovery of a New TeV Gamma-Ray Source: VER J0521+211, ApJ 776, 69 (2013) [arXiv: 1308 . 5017]

[3] Hillas, A. M., Akerlof, C. W., Biller, S. D., et al., The Spectrum of Teravolt Gamma Rays from the Crab Nebula, ApJ 503, 744 (1998)

[4] Shaw, M. S., Romani, R. W., Cotter, G., et al., Spectroscopy of the Largest Ever gamma-Ray-selected BL Lac Sample, ApJ 764, 135 (2013) [arXiv: 1301 . 0323]

[5] Ong, R. A., et al., VERITAS reports a High Gamma-ray Flux from VER J0521+211, The Astronomer's Telegram 2309, 1 (2009)

[6] Krawczynski, H., Hughes, S. B., Horan, D., et al., Multiwavelength Observations of Strong Flares from the TeV Blazar 1ES 1959+650, ApJ 601, 151 (2004) [arXiv:astro-ph/0310158]

[7] Acciari, V. A., Aliu, E., Arlen, T., et al., TeV and Multi-wavelength Observations of Mrk 421 in 2006-2008, ApJ 738, 25 (2011) [arXiv:1106.1210]

[8] Abdo, A. A., Ackermann, M., Ajello, M., et al., Insights into the High-energy gamma-ray Emission of Markarian 501 from Extensive Multifrequency Observations in the Fermi Era, ApJ 727, 129 (2011) [arXiv:1011.5260]

[9] Fomin, V. P., Fennell, S., Lamb, R.C., et al., New methods of atmospheric Cherenkov imaging for gamma-ray astronomy. II. The differential position method, Astroparticle Physics 2, 137 (1994)

[10] Acciari, V. A., Aliu, E., Aune, T., et al., Multiwavelength Observations of a TeV-Flare from W Comae, ApJ 707, 612 (2009) [arXiv:0910.3750]

[11] Li, T.-P. \& Ma, Y.-Q., Analysis methods for results in gamma-ray astronomy, ApJ 272, 317 (1983)

[12] Aleksić, J., Ansoldi, S., Antonelli, L. A., et al., The major upgrade of the MAGIC telescopes, Part I: The hardware improvements and the commissioning of the system, accepted for publication in Astroparticle Physics (2015), arXiv:1409.6073

[13] Aleksić, J., Ansoldi, S., Antonelli, L. A., et al., The major upgrade of the MAGIC telescopes, Part II: A performance study using observations of the Crab Nebula, accepted for publication in Astroparticle Physics (2015), arxiv:1409.5594

[14] Buson, S. et al., Fermi LAT Detection of a Bright GeV Flare from VER 0521+211, The Astronomer's Telegram, 5472, 1 (2013)

[15] Zanin, R., et al., MARS, the MAGIC analysis and reconstruction software, in proceedings of 33rd ICRC (2013), i crc2013-0773 
[16] Aleksić, J., Alvarez, E. A., Antonelli, L. A., et al., Performance of the MAGIC stereo system obtained with Crab Nebula data, Astroparticle Physics 35, 435 (2012) [arXiv: 1108 . 1477]

[17] Burrows, D.N, Hill, J.E., Nousek, J.A., et al., The Swift X-Ray Telescope, Space Science Reviews 120, 165 (2005) [arXiv:astro-ph/0508071]

[18] Stroh, M. C. \& Falcone, A. D., Swift X-Ray Telescope Monitoring of Fermi-LAT Gamma-Ray Sources of Interest, The Astrophysical Journal Supplement 207, 28 (2013) [arXiv : 1305 . 4949] 